\title{
Comparación de los niveles de carga mental y resiliencia que poseen familias con hijos con diversidad funcional a diferencia de familiar con hijos sin diversidad funcional
}

\author{
Natalia Cornejo-Guerra
}

Universidad de Sevilla

\begin{abstract}
Resumen: Diversos autores han analizado las variables resiliencia y carga mental en familias con hijos con diversidad funcional, pero hay escasez de comparativas entre dichas familias y familias con hijos sin diversidad funcional. Por ello, con el objetivo de investigar la posible diferencia entre dichas familias, se ha realizado un estudio cuantitativo, explicativo y transversal. La muestra total está formada por 51 familias (31 con hijos sin diversidad funcional y 20 con hijos con diversidad funcional). Los constructos se evaluaron a través de la Escala de Sobrecarga del Cuidador de Zarit (1980) y la Escala de Resiliencia de Wagnild y Young (1993). Los resultados muestran diferencias estadísticamente significativas entre los dos tipos de familias en la variable carga mental, siendo las familias con hijos con diversidad funcional quienes perciben mayor carga. En relación con la variable resiliencia no se han encontrado diferencias significativas. Finalmente, al valorar la relación entre carga mental y resiliencia dependiendo de tener hijos con/sin diversidad funcional, no se ha encontrado interacción entre dichas variables. En conclusión, resulta necesario ajustar los programas de intervención familiar a las características de los hijos y valorar los factores de protección de los cuidadores para hacer frente a la carga mental.

Palabras clave: Carga Mental, Resiliencia, Cuidadores, Diversidad Funcional.
\end{abstract}

\section{Comparison of the levels of mental load and resilience possessed by families with children with functional diversity compared to families with children without functional diversity}

\begin{abstract}
Several authors have studied the variables resilience and mental burden in families with children with functional diversity, but there is a shortage of comparisons between these families and families with children without functional diversity. Therefore, in order to investigate the possible difference between these families, a quantitative, explanatory and cross-sectional study has been carried out. The total sample consisted of 51 families (31 with children without functional diversity and 20 with children with functional diversity). The constructs were assessed through the Zarit Caregiver Overload Scale (1980) and the Wagnild and Young Resilience Scale (1993). The results show statistically significant differences between the two types of families in the mental burden variable, with families with children with functional diversity being those that perceive the greatest burden. In terms of the resilience variable, no significant differences were found. Finally, when assessing the relationship between mental burden and resilience as a function of having children with or without functional diversity, no interaction was found between these variables. In conclusion, it is necessary to adjust family intervention programs to the characteristics of the children and to assess caregivers' protective factors of to cope with the mental burden.

Keyword: Mental Burden, Resilience, Caregivers, Functional Diversity.
\end{abstract}

\section{AGRADECIMIENTOS}

Transmitir mi más sincero agradecimiento a todas aquellas personas y entidades que han hecho posible la realización de la presente investigación.

Recibido: 19/10/2021 - Aceptado: 11/01/2022 - Avance online: 20/01/2022

*Correspondencia: Natalia Cornejo Guerra

Universidad de Sevilla.

Dirección: 41568, El Rubio, España.

E-mail: ncornejoguerra@gmail.com

Cornejo-Guerra, N. (2022). Comparación de los niveles de carga mental y resiliencia que poseen familias con hijos con diversidad funcional a diferencia de familiar con hiios sin diversidad funcional. Revista de Psicología y Educación, 17(1), 79-96, https://doi.
En primer lugar, a la Universidad, por la enseñanza aportada $y$, en especial, a mis tutores por las orientaciones dadas durante todo el proceso.

Por otro lado, al colegio y a la asociación colaboradores con la investigación, a todas aquellas familias que han querido ser partícipes de esta investigación.

1699-9517/@ 2022 Asociación Científica de Psicología y Educación (ACIPE). Publicado por Consejo General de Colegios Oficiales de Psicólogos, España. Este es un artículo Open Access bajo la CC BY-NC-ND licencia (http://creativecommons.org/licencias/bync-nd/4.0/). 
El cuidado de los hijos, en ocasiones, puede suponer situaciones de estrés que conllevan un desgaste (Rogero, 2010). Este puede ser mayor o menor, dependiendo de las circunstancias de la familia. Es decir, factores como los recursos económicos, el apoyo social, tener un hijo con diversidad funcional, el tipo de diversidad funcional, número de hijos, el trabajo de los cuidadores, entre otros, van a conllevar una mayor o menor intensidad de carga mental (Berastegui y Felpeto, 2016; citado en Vela y Suárez, 2020). Pero żcómo varía la intensidad de esta carga mental y qué factores de protección se observan ante ella?

Cuando se habla del cuidado de los hijos no solo se hace referencia a las tareas de atención y crianza que se realizan, sino también a los componentes invisibles, como la preocupación constante (Eterovic, Mendoza y Sáez, 2015). Es decir, estas diferentes labores y responsabilidades sumadas a las vivencias de preocupación y de vigilancia son las que dan lugar a la sobrecarga (Irazábal, 2016).

Los responsables de esta atención suelen ser padres y madres, $u$, ocasionalmente familiares o personas cercanas. Es decir, cuidadores informales para los cuales dicha tarea puede suponer un reto, debido a la falta de habilidades (Eterovic et al., 2015). A menudo, se establece un cuidador principal, siendo este la víctima del estrés que ocasiona la crianza de los menores.

Pero, ¿̇qué se entiende por carga mental? Zarit, Reever y Bach-Peterson (1986) la definen como el grado en que la persona cuidadora percibe que el cuidado ha influido sobre diferentes aspectos de su salud, su vida social, personal y económica (citado en Cerquera y Pabón, 2016). Por lo tanto, en el caso del cuidado de los hijos, las tareas y responsabilidades de crianza pueden conllevar un estado de estrés que afecte a la salud física y mental, además de problemas socioeconómicos, de tiempo de ocio, de relaciones sociales y de desequilibrio emocional (González et al., 2004; citado en Cerquera y Pabón, 2016).
Ligado a este estrés, se encuentra el sentimiento de competencia y autoeficacia, es decir, cómo de preparados se ven los padres y madres para hacer frente a las diferentes situaciones y asumir las diferentes responsabilidades (Vela y Suárez, 2020). Tal y como Lazarus y Folkman (1984) y Kim et al. (2003) exponían, las estrategias que se posean para hacer frente a la situación van a determinar en mayor o menor medida la sensación de carga percibida (Irazábal, 2016). Pero el tipo de estrategias que sean necesarias pueden variar en función de las características de la persona a cuidar (Vela y Suárez, 2020). Por ejemplo, en el caso de la crianza de un hijo con diversidad funcional, se va a requerir otras habilidades que pueden no ser necesarias para la atención de un menor con desarrollo normativo.

Con respecto al concepto "diversidad funcional", se pretende hacer referencia al constructo, más comúnmente utilizado, "discapacidad". Según la Organización Mundial de la Salud (2020), este último hace referencia a "la interacción entre las personas que tienen algún problema de salud (por ejemplo, parálisis cerebral, síndrome de Down y depresión) y factores personales y ambientales (por ejemplo, actitudes negativas, transporte y edificios públicos inaccesibles y apoyo social limitado)". Debido a las connotaciones negativas que puede tener el término discapacidad, se hará uso del término diversidad funcional, ya que, como destaca Crow (1996; citado en Mañas e Iniesta, 2011) este, según el modelo social crítico, amplía el concepto potenciando la identidad de la persona a través de las capacidades que esta posee.

Así pues, como se venía comentando, la llegada de un hijo con diversidad funcional conlleva una reorganización familiar que puede suponer tensión familiar, desatención parcial de otros hijos y sobreprotección del menor o la menor dependiente (Álvarez et al., 2005; citado en Huerta y Rivera, 2017). Estos cambios en la dinámica familiar suponen altos niveles de estrés que lleva al desgaste del cuidador (Huerta y Rivera, 2017). 
Asimismo, ante los niveles de sobrecarga no solo hay factores que los incrementa, sino que algunos de ellos actúan reduciéndolos, es decir, serían factores de protección frente a la carga mental, como pueden ser el apoyo social (Pérez y Marqués, 2018), ser activo laboralmente (Vela y Suárez, 2020) y la resiliencia (Vela y Suárez, 2020), entre otros.

Por lo tanto, se puede observar que el lugar de trabajo funciona como un espacio para descansar de las responsabilidades y preocupaciones constantes que supone el cuidado de los menores, contribuyendo así la disminución de la carga mental (Joseph y Joseph, 2019; citado en Vela y Suárez, 2020)

Así mismo, la carga mental se ve disminuida gracias al apoyo social, debido al impacto positivo que este ejerce sobre el bienestar psicológico y la calidad de vida, en concreto, el asociacionismo activo. Este, además, actúa promoviendo el tercer factor protector mencionado anteriormente -la resiliencia (Cerquera y Pabón, 2016) ya que contrarresta el impacto sufrido por la demanda de los cuidados (Cerquera y Pabón, 2016), es decir, conforma "una red de seguridad frente a sucesos estresante" (Fernández-Ballestero, 2009; citado en Cerquera y Pabón, 2016, p.36).

Centrándonos en este último factor, el presente estudio se basa en la definición de resiliencia expuesta por Wagnild y Young (1993), quienes argumentan que este constructo es "una característica de la personalidad que modera el efecto negativo del estrés, animando así el proceso de adaptación" (Centeno, 2020, p. 149).

Estos autores, destacan que la variable resiliencia está constituida por cinco dimensiones (Wagnild y Young, 1993): (a) ecuanimidad, entendida como la adopción de una perspectiva equilibrada entre nuestras experiencias y la propia vida; (b) perseverancia, ser persistente ante la adversidad y luchar ante ella; (c) confianza en uno mismo, reconocer las fortalezas propias; (d) comprensión del sentido de la vida, sentir tu propósito en la vida; y (e) soledad existencial, sentirse bien solo, en libertad y con vivencias propias.

Sin embargo, este factor no implica que la persona sea capaz de superar de igual forma toda situación estresante. Es decir, tener una personalidad resiliente no libera de sufrir estrés, pero sí de poder gestionarlo y superarlo con menor impacto (Vela y Suárez, 2020).

De igual forma, como se venía anticipando, frente a esta personalidad resiliente se aprecian algunos factores de protección que ayudan a fomentarla, como puede ser la autoestima, la capacidad resolutiva y el apoyo familiar y social, entre otros (Grotberg, 1995; citado en Cerquera y Pabón, 2016).

Por todo ello, parece que la intensidad de la carga familiar se va a ver afectada, entre otros factores, por la capacidad de resiliencia que los cuidadores posean. Es decir, la destreza que tengan estos para hacer frente a las situaciones adversas (Vela y Suárez, 2020) pueden disminuir el impacto de la sobrecarga. En consecuencia, "ser una persona resiliente es indicador de eficacia en los cuidados realizados, menor agotamiento y afrontamiento positivo" (Navarro, López, Climent y Gómez, 2019, p.270).

En el caso de familias con hijos con diversidad funcional, la adopción de dicha capacidad de resiliencia va a cobrar una gran importancia para poder vivir la experiencia del cuidado con una actitud positiva. Tal y como se señaló anteriormente, la llegada de un hijo con diversidad funcional conlleva una reorganización familiar (Huerta y Rivera, 2017). En este caso, la resiliencia puede funcionar como medio potencial que permite a la familia enfrentarse a la situación a través de sus propios recursos llegando así a una reestructuración adecuada para todos los familiares (Huerta y Rivera, 2017).

Con respecto a esto último, hay estudios en los cuales se reconoce que en cuidadores de personas con diversidad funcional el nivel de 
resiliencia es medio-alto (Cubillos, Gómez, Herrera y Sierra, 2020). Sin embargo, otros autores señalan que estos niveles de resiliencia disminuyen cuando la sobrecarga percibida es mayor (Navarro et al., 2019). En contraposición, la investigación realizada por Vela y Suárez (2020) confirma que la resiliencia actúa como factor protector del estrés que sufren los cuidadoras de menores con diversidad funcional, como ya apuntaban anteriormente otros autores (Eterovic et al., 2015; Suzuki et al.,2018; citado en Vela y Suárez 2020). En sintonía, Speark (2020) en un estudio con madres de niños con TEA encontró que los factores autoconfianza y satisfacción personal del constructo resiliencia sí que tienen un efecto positivo frente a la carga mental. De igual modo, Fernández-Lansac et al. en 2012, encontró que los niveles de resiliencia no solo se asociaban con una menor sobrecarga percibida, sino que también se vinculaban con un apoyo social satisfactorio (Cerquera y Pabón, 2016). Por otro lado, en relación con los factores de riesgo de sufrir sobrecarga, Kim et al. (2017) en un estudio con cuidadores de familiares adultos con discapacidad, observó que, en presencia de discapacidad, la carga psicológica aumentaba. Esto mismo fue hallado por Cuzzocrea et al. (2016; Bujnowska et al., 2021) quienes realizaron una comparativa entre padres de hijos con diversidad funcional y padres de niños con desarrollo típico y obtuvieron que los primeros presentaban niveles más altos de estrés.

Por todo esto, considerando la sobrecarga que implica el cuidado informal de los hijos y, más aún, el de los menores con algún tipo de diversidad funcional, y teniendo en cuenta la actuación de una personalidad resiliente como factor protector, va a ser un punto importante estudiar su relación. Como se ha visto, en estudios precedentes se ha analizado la relación entre ambos constructos. Pero hay escasez de investigaciones en las que se establezca una comparativa entre familias con hijos con diversidad funcional y familias con hijos sin diversidad funcional. Por ello, en la presente investigación se pretende profundizar más en dicha comparación. Todo ello, con el propósito de ayudar a promover un adecuado desarrollo y crianza de los menores, una experiencia positiva de los cuidadores, un ajuste adecuado en la dinámica familiar y un mayor bienestar familiar.

\section{OBJETIVOS}

\section{OBJETIVO GENERAL}

Realizar una comparativa entre los niveles de carga mental y resiliencia que poseen las familias con hijos con diversidad funcional frente a las familias con hijos sin diversidad funcional.

\section{OBJETIVOS ESPECÍFICOS}

1. Analizar los niveles de carga mental en familias con hijos con diversidad funcional y en familias con hijos sin diversidad funcional, conociendo si existe diferencia entre ser cuidador principal o no.

2. Estudiar si la capacidad resiliente varía en función de tener o no hijos con diversidad funcional.

3. Determinar cómo es la relación entre carga mental y resiliencia dependiendo la mediación de tener hijos con o sin diversidad funcional.

\section{MÉTODO}

\section{PARTICIPANTES}

La muestra estudiada en esta investigación es por accesibilidad. La muestra total está compuesta por 51 participantes. Para poder hacer un estudio comparativo se dividió dependiendo de si eran familias con hijos con diversidad funcional o sin diversidad funcional (20 familias con hijo con diversidad funciones y 31 familias con hijo sin diversidad funcional; $38,5 \%$ con diversidad y el $61,5 \%$ sin diversidad). Para asegurar obtener muestra de ambos grupos, se trabajó con usuarios de una asociación de ocio inclusivo para personas con diversidad funcional con sede en 
Sevilla, obteniendo así familias con hijos con diversidad funcional; y familias del alumnado de un colegio de Sevilla para conformar así el grupo control. Para realizar la distinción de los dos grupos, se añadió en el cuestionario la pregunta: ¿̇Tiene hijo con alguna diversidad funcional? A todos los participantes se le administró el mismo cuestionario compuesto por los instrumentos que se describirán a continuación.

Se obtuvo una tasa de respuesta del 100\%. Siendo eliminado un único caso debido a que ante la pregunta "¿Cuántos hijos tiene?" respondió 0 .

Con respecto a las variables sociodemográficas (ver Tabla 1), la mayoría de personas participantes son madres con estudios superiores, de un nivel socioeconómico medio-alto, trabajadoras y que se identifican como cuidadoras principales, teniendo la mayoría más de un hijo.

\section{INSTRUMENTOS}

Los instrumentos de evaluación utilizados fueron autoadministrados en formato online (Anexo I). Estos son los que se señalan a continuación:

- Cuestionario sobre datos sociodemográficos, conformado por preguntas estructuradas y cerradas con diferentes opciones de respuesta sobre características de las familias. Estas son: sexo (masculino, femenino o prefiero no decirlo), nivel de estudio (primaria, secundaria y superiores), situación laboral (activo o desempleo), nivel socioeconómico (tenemos lo mínimo y a veces nos falta; dependemos de nuestro trabajo y llegamos justos a fin de mes; tenemos ingresos suficientes para vivir sin estrecheces; - disfrutamos de una posición holgada que nos permite no tener preocupaciones económicas), con quién convive, número de hijos, si es el cuidador principal de sus hijos, si

Tabla 1

Frecuencia y porcentaje de las variables sociodemográficas de la población estudiada $(N=51)$

\begin{tabular}{|c|c|c|c|}
\hline \multicolumn{2}{|c|}{ Variables sociodemográficas } & \multirow{2}{*}{$\begin{array}{c}\text { Frecuencia } \\
44\end{array}$} & \multirow{2}{*}{$\begin{array}{c}\text { Porcentaje } \\
86.3\end{array}$} \\
\hline Sexo & Femenino & & \\
\hline & Masculino & 7 & 13.7 \\
\hline \multirow{3}{*}{$\begin{array}{l}\text { Nivel de estudio } \\
\text { Nivel de estudio }\end{array}$} & Primaria & 2 & 3.9 \\
\hline & Secundaria & 6 & 11.8 \\
\hline & Superiores & 43 & 84.3 \\
\hline \multirow[t]{2}{*}{ Situación laboral } & Desempleo & 13 & 25.5 \\
\hline & Activo & 38 & 74.5 \\
\hline \multirow[t]{4}{*}{$\begin{array}{l}\text { Nivel } \\
\text { socioeconómico }\end{array}$} & Tenemos lo mínimo y a veces nos falta & 2 & 3.9 \\
\hline & $\begin{array}{l}\text { Dependemos de nuestro trabajo y llegamos justos } \\
\qquad \text { a fin de mes }\end{array}$ & 6 & 11.8 \\
\hline & $\begin{array}{c}\text { Tenemos ingresos suficientes para vivir sin } \\
\text { estrecheces }\end{array}$ & 33 & 64.7 \\
\hline & Disfrutamos de una posición holgada & 10 & 19.6 \\
\hline
\end{tabular}


Tabla 1 (Continuación)

Frecuencia y porcentaje de las variables sociodemográficas de la población estudiada $(N=51)$

\begin{tabular}{|c|c|c|c|}
\hline \multicolumn{2}{|c|}{ Variables sociodemográficas } & Frecuencia & Porcentaje \\
\hline \multirow{2}{*}{ Número de hijos } & 1 & 15 & 29.4 \\
\cline { 2 - 4 } & 2 & 23 & 45.1 \\
\cline { 2 - 4 } & 3 & 10 & 19.6 \\
\cline { 2 - 4 } & 4 & 3 & 5.9 \\
\hline \multirow{2}{*}{ Cuidador principal } & No & 6 & 11.8 \\
\cline { 2 - 4 } & Sí & 45 & 88.2 \\
\hline
\end{tabular}

sus hijos tiene diversidad funcional y si alguna de las personas con las que convive es una persona dependiente.

- Escala de Sobrecarga Mental del Cuidador de Zarit en su adaptación española (Martín et al., 1996) a través de la cual se obtiene una puntuación subjetiva del grado de sobrecarga que tiene el cuidador de una persona dependiente (Larrinoa et al., 2011). La escala está conformada por 22 ítems y es de tipo Likert con cinco opciones, desde nunca (1) a casi siempre (5), pudiendo obtener así un resultado total entre 22 y 110 puntos. Esta puntuación representa la carga mental del cuidador, considerando la existencia de sobrecarga a partir de 56 puntos (Breinbaver et al., 2009).

Se eligió dicha escala debido a que ha sido empleada para otras investigaciones previas en familias o en cuidadores de personas dependientes (Eterovic et al., 2015; Navarro et al., 2019; Salazar et al., 2020; Seperak, 2016), asemejándose sus objetivos al del presente estudio.

En la redacción original de los ítems del instrumento se preguntaba por "su familiar" siendo modificado en esta investigación por "su hijo", debido a que el objetivo del estudio es evaluar la sobrecarga que padece el cuidador por el cuidado de su/s hijo/s. Además, de dicha modificación también se hizo un cambio en el ítem "¿Siente que ha perdido el control de su vida desde que comenzó la enfermedad de su familiar?" por "¿̇iente que ha perdido el control de su vida desde que nació su hijo?". A pesar de ello, el Alfa de Cronbach obtenido para esta escala fue de .89 -a diferencia de la prueba original que obtuvo .91 (Larrinoa et al., 2011) - por lo que las modificaciones no han afectado a la fiabilidad del instrumento.

- Escala de Resiliencia de Wagnild y Young (1993) en la adaptación de Castilla, Rodríguez, Shimabukuro, Valdivia y TorresCalderón (2014) para evaluar, a través de un cuestionario autoadministrado, el nivel de resiliencia de los padres y madres que participan en el estudio. Este está diseñado para la evaluación de adultos y adolescentes (Wagnild y Young, 1993). El instrumento consta de 25 ítems, siendo una escala tipo Likert de siete alternativas que oscilan entre 1 (totalmente en desacuerdo) y 7 (totalmente de acuerdo). Pudiendo variar así, el nivel de resiliencia, entre 25 y 175 puntos. Se considerará baja cuando se obtiene una puntuación inferior a 70 y alta cuando esta es mayor que 78 (Castro, 2018).

Como se comentó al comienzo, el constructo resiliencia, según Wagnild y Young (1993) consta de cinco características (ecuanimidad, perseverancia, confianza en 
sí mismo, satisfacción y sentirse bien solo). Sin embargo, posteriormente, a la hora de evaluarla, se habló de dos factores (Wagnild y Young, 1993; citado en Castilla, Coronel, Bonilla, Mendoza y Barboza, 2016). El primer factor, competencia personal, engloba a la confianza, independencia, determinación, control, ingenio y perseverancia (ítems: 1, 2, $3,4,5,6,9,10,13,14,15,17,18,19$, 20,23 y 24); y el factor de adaptación de uno mismo y de la vida, que hace referencia a la capacidad de adaptación, equilibrio, flexibilidad y perspectiva de vida equilibrada (ítem: 7, 8, 11, 12, 16, 21, 22 y 25).

De igual modo, este instrumento ha sido usado por autores que tenían como objetivo evaluar la resiliencia en muestras de cuidadores de personas dependientes (Centeno, 2020; Navarro et al., 2019; Seperak, 2016; Ponce y Torrecillas, 2017).

En este estudio, se ha aplicado la adaptación al español, realizada por Castilla et al. (2014), en la cual se obtuvo un coeficiente Alfa de Cronbach de .89 para la escala total, de .91 para el Factor I y de .81 para el Factor II (Castilla et al.,2016). En la presente investigación este coeficiente ha sido de .878 .

\section{PROCEDIMIENTOS}

El muestreo empleado fue no probabilístico, este fue por accesibilidad. Siendo la única condición que fueran padres/madres y que sus hijos fueran alumnos del colegio o participantes de la asociación nombrados anteriormente.

En un primer lugar, se contactó con ambos grupos, de forma indirecta. En el caso del colegio se informó a la orientadora promotora del Aula de Familia y a través de un correo electrónico se le envió el cuestionario para que lo pudieran realizar vía online. Y, por otro lado, la directora de la asociación se encargó de anunciárselo a las diferentes familias, poniendo a su disposición la posibilidad de ser partícipes del presente estudio realizando, de igual forma, el cuestionario vía online.
En dicho cuestionario autoadministrado (Anexo I) se incluyó una breve explicación sobre en qué consistía su colaboración -intentando que esta sesgara lo mínimo la respuesta de los participantes -, el anonimato de sus respuestas y la garantía de ser guardada la confidencialidad de los datos que se brinda. Finalmente, se les indicó que al enviar el cuestionario estarían aceptando ser partícipes del estudio.

Para la realización de dicho cuestionario se dio el plazo de dos semanas, para que pudiera elegir el momento y el día en el que tuvieran disponibilidad para cumplimentarlo. Se estima que el tiempo necesario para responder por completo al cuestionario fue de 20 minutos.

En un primer lugar, tras darse las pautas nombradas anteriormente, se les preguntó algunas características sociodemográficas (sexo, nivel de estudio, situación laboral, nivel socioeconómico, convivientes, número de hijos, cuidador principal, si tienen hijos con diversidad funcional $u$ otra persona dependiente con la que conviva). Seguidamente, debían respondes a ambos instrumentos tras leer unas breves instrucciones, administrándose primero la Escala de Sobrecarga del cuidador de Zarit en su adaptación española (Martín et al., 1996) y, finalmente, el Cuestionario sobre Resiliencia de Wagnild y Young (1993) en la adaptación de Castilla et al. (2014). En total, debían aportar 7 datos sociodemográficos y responder 47 ítems, siendo obligatoria la respuesta a cada uno de ellos.

Transcurrido el plazo de las dos semanas, se cerró el cuestionario online y se recopiló la información en una base de datos. Para realizar el análisis de estos, se utilizó el paquete estadístico SPSS versión 26.0. A través de este se realizaron análisis de comparación de medias y regresión con interacción (véase Tabla 2). Asimismo, para la evaluación del tamaño de efecto se emplearon los estándares de Cohen (1988) -pequeño, medio o grande De igual forma, para el cálculo de la potencia estadística de los diferentes análisis se empleó el programa G*Power (Faul, Erdfelder, Buchner y Lang, 2009). 
Tabla 2

Relación del tipo de análisis de datos según el objetivo específico

\begin{tabular}{|c|c|}
\hline OBJETIVO ESPECÍFICO & TIPO DE ANÁLISIS \\
\hline $\begin{array}{l}\text { 1. Analizar los niveles de carga mental en familias con hijos } \\
\text { con diversidad funcional y en familias con hijos sin diversidad } \\
\text { funcional. }\end{array}$ & $\begin{array}{l}\text { Análisis de comparación de medias a través de la prueba t de } \\
\qquad \text { Student. }\end{array}$ \\
\hline $\begin{array}{l}\text { 2. Estudiar si la capacidad resiliente varía en función de tener o } \\
\qquad \text { no hijos con diversidad funcional. }\end{array}$ & $\begin{array}{l}\text { Análisis de comparación de medias a través de la prueba t de } \\
\qquad \text { Student }\end{array}$ \\
\hline $\begin{array}{l}\text { 3. Determinar cómo es la relación entre carga mental y } \\
\text { resiliencia dependiendo de tener hijos con o sin diversidad } \\
\text { funcional. }\end{array}$ & $\begin{array}{c}\text { Análisis de la regresión con interacción a través de un diagrama } \\
\text { de dispersión y del estudio de la regresión entre carga mental } \\
\text { y resiliencia, con esta última como predictora y con los } \\
\text { participantes segmentados según la variable moderadora - } \\
\text { tener hijos con/sin diversidad funcional-. }\end{array}$ \\
\hline
\end{tabular}

Por todo ello, se puede decir que el estudio que se llevó a cabo es una investigación de campo de tipo explicativa. Además, el diseño establecido para la recogida de datos fue transversal, recogiendo los datos en un único momento. En suma, se considera que la estructura del objeto de estudio de la presente investigación es relacional.

\section{RESULTADOS}

A continuación, se muestra el análisis de las relaciones existentes entre las variables carga mental, resiliencia e hijos con diversidad funcional. Por lo general, los resultados indican que las familias participes poseen una baja carga mental $(M=51.53 ; D T=13.71)$ y una personalidad resiliente $(M=144.94$; $D T=14.59)$.

En primer lugar, se ha realizado la prueba $\dagger$ de Student, debido a que se cumplía el supuesto de normalidad, con el objetivo de contrastar si existen diferencias entre familias con hijos con diversidad funcional y familias con hijos sin diversidad funcional en los niveles de carga mental. Según la prueba de Levene no hay homogeneidad de las muestras $(p=.04)$, por lo cual no se asumen las varianzas iguales. Los resultados muestran que sí existen diferencias estadísticamente significativas entre los grupos $t(32.22)=-2.99, p<.05$. Estos indican que las familias con hijos con diversidad funcional $(M=58.55 ; D T=14.81)$ presentan mayor sobrecarga mental que las familias con hijos sin diversidad funcional $(M=47$; DT $=$ 10.95) con un intervalo de confianza al $95 \%$ entre -19.39 y -3.70 y un tamaño de efecto grande $(d=.92)$, siguiendo los estándares de Cohen (1988). Además, observando el grado de significación y el tamaño de efecto, ambos apuntan que el efecto probablemente existe a nivel poblacional (Jiménez y Trigo, 2015).

Asimismo, el tamaño muestral utilizado, $N=51$, ha permitido alcanzar una potencia estadística de $1-\beta=.87$. Dicha potencia fue calculada mediante el uso de G*Power 3.1. (Faul et al., 2009) para el análisis expuesto anteriormente, con $\alpha=.05$.

Si además de estudiar la carga mental en relación con tener hijo/s con/sin diversidad funcional, se identifica en cada grupo quiénes son las personas participantes que se reconocen como cuidadores principales, se observa que el incremento de sobrecarga es aún mayor en el caso de ser cuidador principal con hijo/s con diversidad funcional (ver Figura 1). 


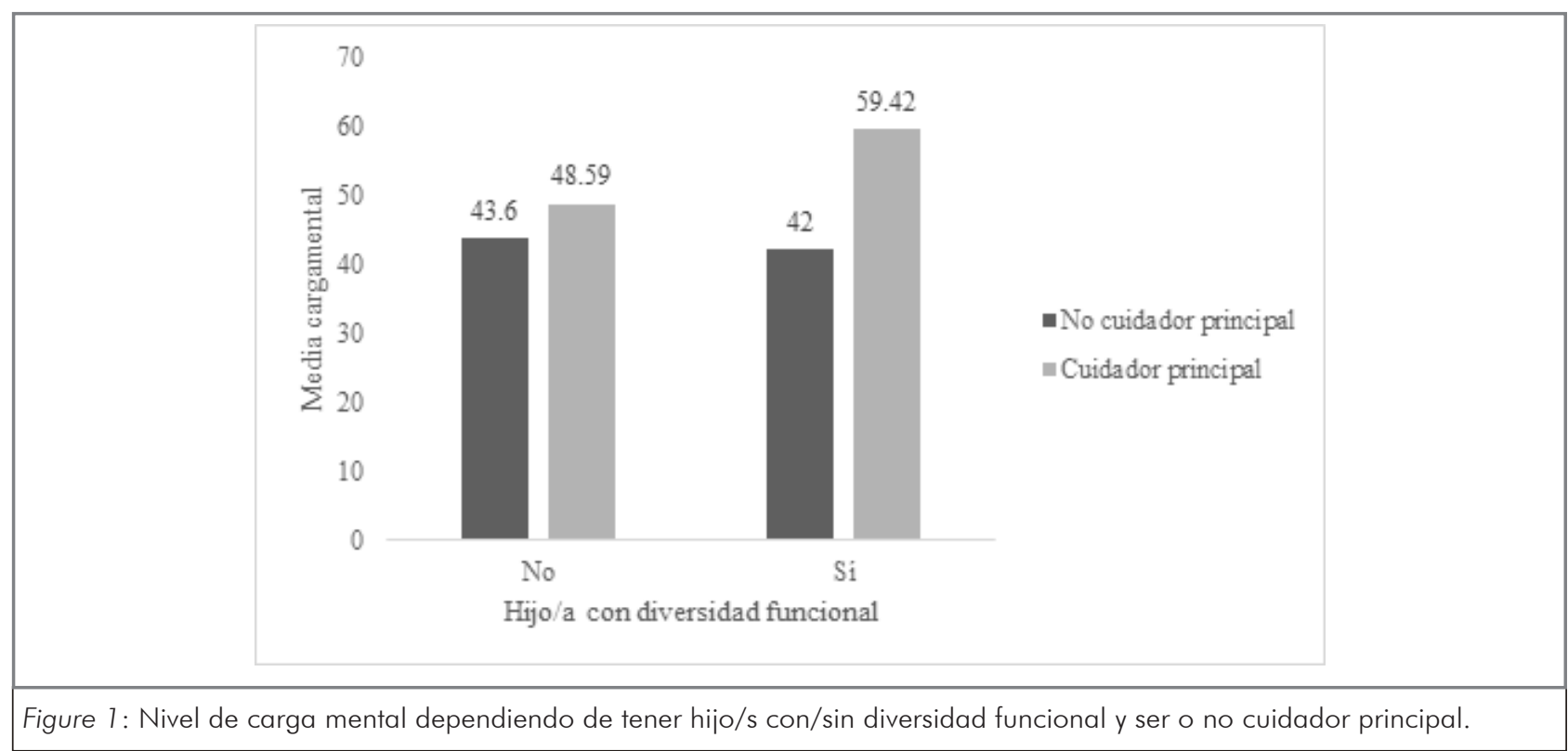

\section{Tabla 3}

Prueba $t$ de Student y tamaño de efecto para los factores de resiliencia dependiendo de tener hijos con/sin diversidad funcional $(N=51)$.

\begin{tabular}{|c|c|c|c|c|c|c|}
\hline & $M$ & $D T$ & $t$ & $g l$ & $p$ & $d$ \\
\hline F1. Competencia personal & 99.86 & 10.37 & -.51 & 42.25 & .61 & .15 \\
\hline $\begin{array}{c}\text { F2. Adaptación de uno mismo y de } \\
\text { la vida }\end{array}$ & 45.07 & 4.91 & -1.39 & 43.18 & .17 & .39 \\
\hline
\end{tabular}

En segundo lugar, a pesar de que no se cumplía el supuesto de normalidad en el estudio del segundo objetivo -análisis de la relación de resiliencia y el hecho de tener hijos con/sin diversidad funcional -, se ha usado, de igual forma, la prueba t de Student, debido su robustez. En este caso, no se ha encontrado diferencias significativas $p>.05$. Es decir, la capacidad de resiliencia en el grupo de familias con hijos con diversidad funcional $(M=144.06$; $D T=14.77)$ no es significativamente diferente a la de las familias con hijos sin diversidad funcional $(M=147.45$; $D T=14.83)$, con un intervalo de confianza al $95 \%$ entre -11.61 y 4.84 . El tamaño de efecto encontrado en este caso, según los niveles convencionales de Cohen (1988) es pequeño $(d=.24)$, por lo tanto, estos dos datos señalan que el efecto probablemente no existe a nivel poblacional (Jiménez y Trigos, 2015).
En este caso, la potencia estadística alcanzada $(N=51)$ es $1-\beta=.20$, con $\alpha$ $=.05$, calculada de igual modo a través del programa G*Power 3.1. (Faul et al., 2009).

Asimismo, si se realiza este mismo análisis, pero diferenciando entre los dos factores de los cuales se compone la resiliencia-competencia personal y adaptación de uno mismo y de la vida (Wagnild y Young, 1993; citado en Castilla et al. 2016) -, se observa de igual forma que la puntuación alcanzada en estos en el grupo de padres y madres con hijos con diversidad funcional no es significativamente diferente a la puntuación del grupo control con un tamaño de efecto pequeño en ambos factores (véase Tabla 3).

Finalmente, con el objetivo de valorar la relación existente entre resiliencia y carga 


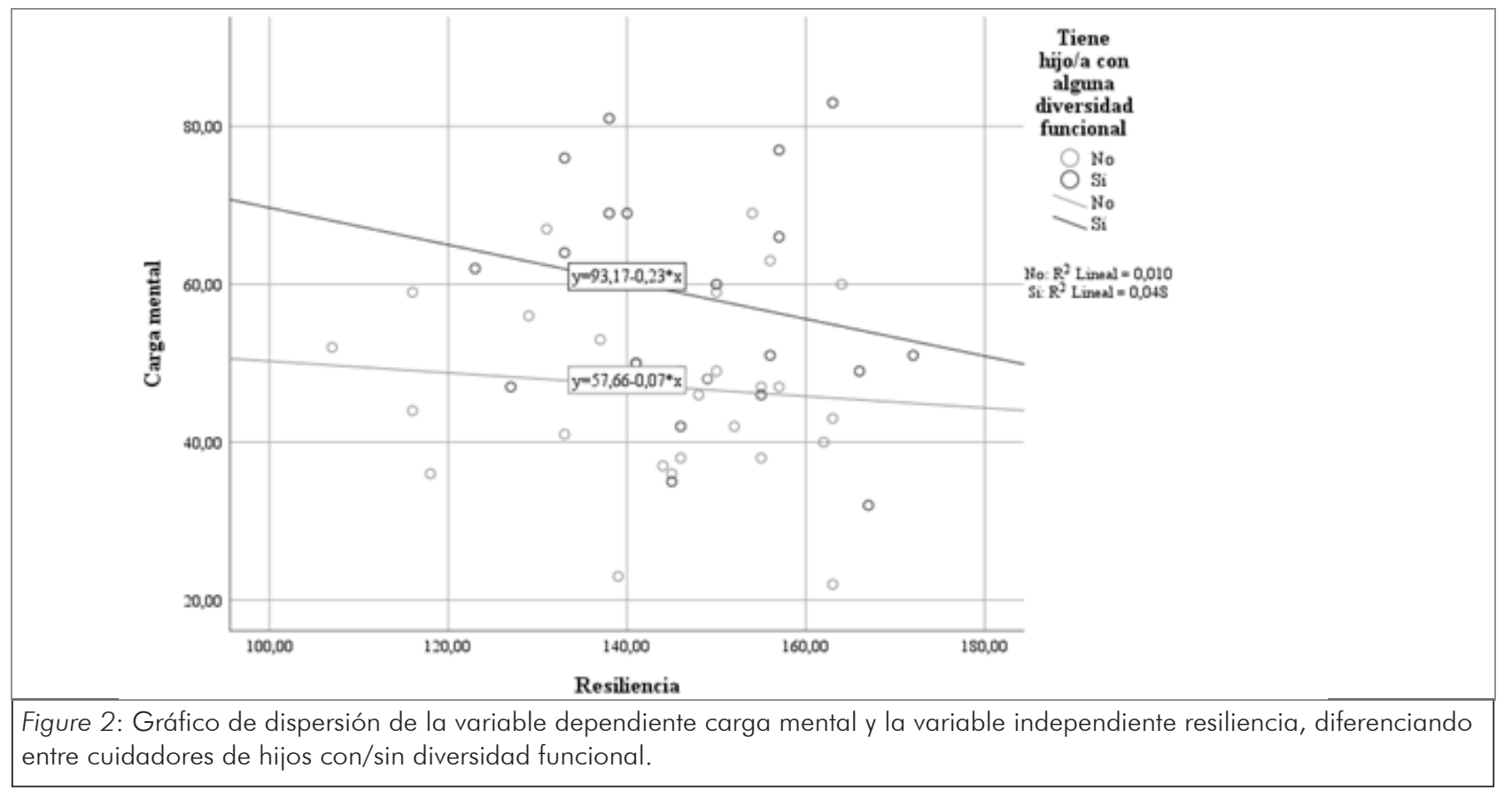

mental, teniendo en cuenta cómo influye el hecho de tener un hijo con diversidad funcional en dicha relación, se realizó un gráfico de dispersión (ver Figura 2). Como puede observarse, los niveles de carga mental disminuyen cuando la capacidad de resiliencia aumenta, encontrando mayor pendiente en el grupo de familias con hijos con diversidad funcional $\left(R^{2}=.048\right)$.

Con el fin de conocer si dicha relación es significativa se realizó un análisis de interacción con regresión con la variable carga mental como dependiente, resiliencia como variable independiente y como variable moderadora tener hijos con/sin diversidad funcional 10 para hijos sin diversidad funcional y 1 para hijos con diversidad funcional).

Tras analizar los supuestos de normalidad y homocedasticidad y comprobar que se cumplen, se encontró una ecuación de regresión significativa $(F(3.47)=3.86, p<.05$, con un $\left.R^{2}=.198\right)$. Obteniéndose que .074 es la disminución del nivel de carga mental por cada unidad que aumenta la capacidad de resiliencia, todo ello en ausencia de hijos con diversidad funcional. En presencia de hijos con diversidad funcional, la disminución de la sobrecarga es de 12.13, cuando existe una capacidad de resiliencia media $(M=$
145.39). En suma, el cambio de efecto de los niveles de carga mental en presencia de hijos con diversidad funcional es de -. 16. Por lo tanto, en dicho caso, el efecto es de -.23. Sin embargo, la interacción de la variable resiliencia con la variable moderadora no es significativa $(p=.54)$.

No obstante, para saber si el efecto de este último coeficiente $(B=-.23)$ es significativo, se realiza un análisis de regresión lineal con los datos segmentados en los dos grupos -familias con hijos con diversidad funcional y familias con hijos sin diversidad funcional (ver Tabla 4). Como puede verse, la relación existente entre carga mental y resiliencia, moderada por tener hijos con/sin diversidad funcional, no es estadísticamente significativa.

Asimismo, la potencia estadística alcanzada $(N=51)$, para un tamaño de efecto mediano $\left(f^{2}=.25\right)$ según los estándares de Cohen (1988), es $1-\beta=.83$, con $\alpha=.05$, calculada de igual modo a través del programa $G^{*}$ Power 3.1. (Faul et al., 2009).

\section{DISCUSIÓN}

El objetivo principal de este estudio era analizar las variables carga mental y resiliencia a través de la comparación de dos grupos 


\section{Tabla 4}

Prueba $t$ de Student y tamaño de efecto para los factores de resiliencia dependiendo de tener hijos con/sin diversidad funcional ( $N=51)$.

\begin{tabular}{|c|c|c|c|c|}
\hline & $\beta$ & $\rho$ & $R^{2}$ & $\begin{array}{c}\text { Error estándar de } \\
\text { estimación }\end{array}$ \\
\hline $\begin{array}{c}\text { Hijo/s sin diversidad } \\
\text { funcional }\end{array}$ & -.10 & .59 & .01 & 11.08 \\
\hline $\begin{array}{c}\text { Hijo/s con diversidad } \\
\text { funcional }\end{array}$ & -.22 & .35 & .04 & 14.85 \\
\hline
\end{tabular}

(familias con hijos con diversidad funcional y familias con hijos sin diversidad funcional). Con los resultados obtenidos se ha podido comprobar la diferencia que presentan estos grupos en dichas variables y ver cómo influye tener hijos con diversidad funcional en la relación entre carga mental y resiliencia.

A diferencia de estudios precedentes -en los cuales el perfil sociodemográfico de cuidadores informal estudiado es de mujeres con estudios primarios y amas de casa (Blanco et al., 2007; Coira y Bailón, 2014; Gálvez et al., 2013; García et al., 2004; Larrinoa et al., 2011 ; Rivera et al., 2009; citado en Navarro et al., 2019) -, en la presente investigación los resultados se establecen sobre un perfil, en su mayoría, de mujeres con estudios superiores y activas laboralmente.

En primer lugar, con respecto al primer objetivo -analizar los niveles de sobrecarga en familias con hijos con diversidad funcional y en familias con hijos sin diversidad funcional - los resultados apoyaban que la carga mental en el caso de familias con hijos con diversidad funcional es más elevada. Siendo más evidente esta diferencia al establecer la comparativa entre cuidadores principales y no principales, puesto que los cuidadores principales son los que asumen todas las responsabilidades y las víctimas del estrés que genera la crianza de los menores (Perlado, 1995; Larrinoa et al., 2011 ). Estos coinciden con las conclusiones en estudios previos como las de Kim (2017), quien afirma que la carga psicológica aumenta en presencia de diversidad funcional, o las de Cuzzocrea et al. (2016; Bujnowska et al., 2021) quienes concluyeron en su comparativa que los padres de hijos con diversidad funcional presentaban niveles más altos de estrés.

Sin embargo, cabe destacar que los niveles de carga mental encontrados, en ambos grupos, no son elevados. Esto es, a pesar de que haya diferencia en las medias de ambos tipos de familia, estas indican que no hay sobrecarga mental. Esto se puede deber, a que tanto las familias procedentes del colegio, que forman parte del Aula de Familia -grupo control -, como las familias pertenecientes a la asociación -familias con hijos con diversidad funcional- cuentan con apoyo social, que tal y como se mencionó al principio, es un factor protector frente a dicha variable (Pérez y Marqués, 2018). Asimismo, el estudio realizado por Navarro et al. (2019) apoya que el sentimiento de pertenencia, es decir, el asociacionismo, puede conllevar una disminución de la carga percibida.

Además, como se comentó anteriormente, la población estudiada es, en su mayoría, activa laboralmente, siendo un factor que puede haber actuado como amortiguador del estrés. Ya que hay estudios que apoyan que el trabajo funciona como un espacio 
de descanso de las labores como cuidador (Joseph y Joseph, 2019; citado en Vela y Suárez, 2020).

De igual modo, la variable resiliencia también actúa atenuando los niveles de la sobrecarga (Vela y Suárez, 2020) y, en el caso del presente estudio, se ha encontrado que los participantes poseen altos niveles de capacidad de resiliencia, lo cual podría explicar los niveles de carga mental encontrados.

En consonancia con esto último, el segundo objetivo que se pretendía estudiar era conocer si la posesión de capacidad de resiliencia varía en función de tener o no hijos con diversidad funcional. Los resultados obtenidos apuntan que no existen diferencias significativas en los niveles de resiliencia que poseen ambos grupos. Esto es, tener un hijo con diversidad funcional no supone desarrollar mayor o menor capacidad de resiliencia. Asimismo, el tamaño de efecto encontrado es pequeño, por lo que se podría afirmar que dicha relación probablemente no exista a nivel poblacional (Jiménez y Trigo, 2015).

No obstante, Centeno (2020) en un estudio con cuidadores de personas con discapacidad intelectual leve y moderada, obtuvo que cuando la discapacidad es moderada, al demandar mayor cuidado, los niveles de resiliencia disminuyen.

A pesar de ello, en este caso, los niveles de resiliencia encontrados son altos en ambos grupos. Según Huerta y Rivera (2017) sobre ello puede influir que son familias con una red de apoyo fuerte y estable. Esto mismo se afirma en un estudio de Fernández-Lansac et al. (2012), en el cual se encontró que los mayores niveles de resiliencia se asociaban con una menor sobrecarga subjetiva de los participantes y una mayor satisfacción con el apoyo social recibido (citado en Cerquera y Pabón, 2016). Por lo tanto, la participación de las familias en grupos de apoyo como son las asociaciones o aulas de familias, puede haber fomentado su capacidad de resiliencia.
Finalmente, con respecto al último de los objetivos, se conocen diversos estudios en los cuales se ha observado una relación significativa y negativa entre carga mental y resiliencia (Navarro et al., 2019; Seperak, 2016; Vela y Suárez, 2020). Por ello, se pretendía profundizar más y estudiar si en dicha relación influye el hecho de tener un hijo con diversidad funcional.

En la población estudiada se ha encontrado que dicha variable no interacciona en la relación entre estos constructos. Es decir, a pesar de que tener un hijo con diversidad funcional sí influye en la carga mental percibida por los cuidadores y que según autores como Navarro et al. (2019), Seperak (2016) y Vela y Suárez (2020), si existe una relación entre carga mental y resiliencia, tener un hijo con diversidad funcional no interacciona en dicha dependencia.

\section{LIMITACIONES}

En el desarrollo de esta investigación se ha encontrado varias limitaciones. En primer lugar, el muestreo empleado para la población estudiada ha sido no probabilístico. Además, la muestra es pequeña y al ser dividida para el análisis aún más, por lo que la generalización de los resultados es débil. Así mismo, está conformada, en su mayoría, por mujeres con un alto nivel educativo y con un estatus socioeconómico medio-alto, lo que implica que no sea representativa. En suma, se ha empleado un grupo reducido de participantes, lo que ha podido afectar al análisis estadístico. Por ello, sería necesario, en futuros estudios, analizar una muestra más grande y con variables sociodemográficas que permita obtener resultados más generalizables.

En relación con el tamaño muestral, otra limitación a considerar es la baja potencia estadística obtenida en el análisis del segundo objetivo, lo cual puede haber afectado a los resultados encontrados. Debido a ello, como mejora se debería comprobar la muestra que se necesita a priori, para obtener una mayor potencia estadística. 
Considerando las diferentes variables sociodemográficas registradas (género, nivel socioeconómico, nivel de estudio, situación laboral, número de hijos...), no haberlas incluido en el análisis puede suponer una limitación del estudio, ya que podría aportar datos relevantes. Luego, en futuros estudios puede ser importante atender a este tipo de factores.

Finalmente, otro punto a tener en cuenta es la falta de literatura para contrastar los resultados obtenidos. Hay una escasez de estudios en la que se comparen familias con hijos con diversidad funcional y familias con hijos sin diversidad funcional; los estudios se centran en mayor medida en el primer grupo sin hacer un análisis comparativo. Asimismo, a pesar de que hay numerosas investigaciones sobre la relación entre resiliencia y carga mental, no se suele incluir la posible interacción que puede sufrir esta al incluir la variable "hijos con diversidad funcional". Por ello, como propuesta para futuros estudios, sería interesante seguir profundizando en dicha comparativa para conocer aún más sobre cómo afecta a la dinámica familiar el hecho de tener hijos con diversidad funcional.

\section{CONCLUSIÓN}

Tener un hijo/ puede suponer un desafío, nuevas responsabilidades y preocupaciones constantes, pero gracias a investigaciones como esta se puede encontrar factores que ayuden a conseguir un bienestar familiar y un desarrollo adecuado de los menores.

Estos cuidados no siempre van a suponer los mismos niveles de carga mental, como se ha podido comprobar, características como que el menor presente algún tipo de diversidad funcional puede influir a que dichos niveles se vean aumentados. Sin embargo, frente a estos se observan factores como la resiliencia, el apoyo social o ser activo laboralmente que ayudan a su disminución.

Por ello, una de las conclusiones más destacadas es la importancia que tiene el asociacionismo. Esto es, el sentimiento de pertenencia a un grupo y el apoyo de las personas que lo conforman puede ayudar a hacer frente al desafío. Asimismo, se observa que dicho factor fomenta el desarrollo de una personalidad resiliente, siendo otro factor amortiguador del desgaste sufrido.

En suma, para realizar una adecuada planificación de programas de educación parental va a ser importante tener en cuenta si los menores tienen diversidad funcional, pudiendo así ajustar las pautas a las características de la persona a cuidar. Además, dichas intervenciones familiares deberán considerar los factores de protección de los cuidadores principales para hacer frente a la carga mental que supone la crianza de los menores.

\section{- Conflicto de intereses.}

Los autores declaran no tener conflicto de intereses.

\section{REFERENCIAS}

Breinbaver, H., Vásquez, H., Mayanz, S., Guerra, C. y Millán, T. (2009). Validación en Chile de la Escala de Sobrecarga del Cuidador de Zarit en sus versiones original y abreviada. Rev Med Chile, 137, 657-665.

Bujnowska, A.M., Rodríguez, C., García, T., Areces, D. y Marsh, N.V. (2021). Coping with stress in parents of children with developmental disabilities. International Journal of Clinical and Health Psychology, 21 (3). https://doi.org/10.1016/i. iichp.2021.100254

Castilla, H., Coronel, J., Bonilla, A., Mendoza, M. y Barboza, M. (2016). Validez confiabilidad de la Escala de Resiliencia (Scale Resilience) en una muestra de estudiantes y adultos de la Ciudad de Lima. Revista Peruana de Psicología y Trabajo Social, 4(2), 121 $136 . \quad$ https://repositorio.upn.edu.pe/ handle/1 1537/26829

Castilla, H., Rodríguez, T. C., Shimabukuro, M., Valdivia, A., y Torres-Calderón, M. F. (2014). Análisis exploratorio de la escala de resiliencia de Wagnild y Young en una muestra de adolescentes, jóvenes y adultos 
universitarios de Lima Metropolitana. Revista psicología Arequipa, 4(1), 80-93.

Castro C., K. P. (2018). Propiedades psicométricas de la escala de resilencia de Wagnild y Young en adolescentes de Pachacutec, Ventanilla. [Trabajo de titulación, Universidad César Vallejo]. https://repositorio.ucv.edu.pe/ handle/20.500.12692/29971

Centeno Túqueres, M. J. (2020). Resiliencia en cuidadores primarios de personas con discapacidad intelectual leve y moderada pertenecientes a la Fundación de Enseñanza Individual para Niños, Niñas y Adolescentes (EINA). [Trabajo de titulación, Universidad Central del Ecuador]. http://www.dspace.uce.edu.ec/ handle/25000/20986

Cerquera, A. M., y Pabón, D. K. (2016). Resiliencia y variables asociadas en cuidadores informales de pacientes con Alzheimer. Revista Colombiana de Psicología, 25(1), 33-46. https://doi. org/10.15446/rcp.v25n 1.44558

Cohen, J. (1988). Statistical power analysis for the behacioral sciences ( $2^{a}$ ed.). Hillsdale, NJ: Lawrence Erlbaum Associates.

Cubillos, K., Gómez, M., Herrera, M., y Sierra, M (2020). Sobrecarga, resiliencia y estilos de apego en cuidadores de niños con cáncer. [Trabajo grado de Psicología, Universidad de San Buenaventura Colombia]. http://bibliotecavirtualoducal. uc.cl:8081/handle/10819/7770

Eterovic, C. A., Mendoza Parra, S. E., y Sáez Carrillo, K. L. (2015). Habilidad de cuidado y nivel de sobrecarga en cuidadoras/ es informales de personas dependientes. Enfermería Global, 14(2), 235. https:// doi.org/10.6018/eglobal.14.2.198121

Faul, F., Erdfelder, E., Buchner, A. y Lang, A. G. (2009). Statistical power analyses using G*Power 3.1: Tests for correlation and regression analyses. Behavior Research Methods, 41, 1149-1160. http://www. qpower.hhu.de/

Huerta Ramírez, Y., y Rivera Heredia, M. E. (2017). Resiliencia, recursos familiares y espirituales en cuidadores de niños con discapacidad. Journal of Behavior, Health \& Social Issues, 9(2), 70-81. https://doi. org/10.1016/i.jbhsi.2018.01.005
IBM Corp. Publicado en 2019. IBM SPSS Statistics para Windows, versión 26.0. Armonk, Nueva York: IBM Corp.

IrazábalGiménez, M. (2016). La carga familiar de los cuidadores de personas jóvenes y adultas diagnosticadas de discapacidad intelectual y trastorno mental: una revisión sistemática. Psiquiatría Biológica, 23(3), 93-102. https://doi.org/10.1016/i. psiq.2016.08.004

Jiménez, L. y Trigo, M.E. (2015) Guía para la elaboración del informe final. Trabajo fin de grado en psicología. Sevilla: Iris-Copy. https://personal.us.es/trigo/materiales/ JimenezTrigo2015GUIAinforme.pdf

Kim, D. (2017). Relationship between caregiving stress depression, and self-esteem in family caregivers of adults with disability. Occupational Therapy International. Occupational Therapy International. https://doi. org/10.1155/2017/1686143

Larrinoa, P. F. de L., Rodríguez, S. M., Marqués, N. O., Zabaleta, M. C., Eizaguirre, J. S., y Marroquín, I. G. (2011). Autopercepción del estado de salud en familiares cuidadores y su relación con el nivel de sobrecarga. Psicothema, 23(3). https://www.redalyc.org/articulo. oa? id $=72718925008$

Mañas Viejo, C., e Iniesta Martínez, A. (2011). Diversidad funcional género y educación. International Journal of Developmental and Educational Psychology: INFAD. Revista de Psicología, 7 (4), 353-362. https://dialnet.unirioja.es/ servlet/articulo? codigo $=5641336$

Martín M, Salvadó I, Nadal S, Miji LC, Rico JM, Lanz P y Tausig, M.I. (1996) Adaptación para nuestro medio de la Escala de Sobrecarga del Cuidador (Caregiver Burden Interview) de Zarit. Revista de gerontología, 6(1), 338-46.

Navarro-Abal, Y., López-López, M. J., Climent-Rodríguez, J. A., y GómezSalgado, J. (2019). Sobrecarga, empatía y resiliencia en cuidadores de personas dependientes. Gaceta Sanitaria, 33(3), 268-271. https://doi.org/10.1016/i. gaceta.2017.11.009

Organización Mundial de la Salud (OMS). (2020). Centro de prensa: Discapacidad 
y salud. Web oficial de la Organización Mundial de la Salud. Disponible en: http:// www.who.int/mediacentre/factsheets/ fs352/es/

Pérez, J. J. N., y Marqués, A. C. (2018). Sobrecarga familiar, apoyo social y salud comunitaria en cuidadores de personas con trastorno mental grave. Revista da Escola de Enfermagem Da USP, 52. https://doi.org/10.1590/s1980220x2017029403351

Ponce, J. E., y Torrecillas, A. M. (2017). Estudio de factores resilientes en familiares de personas con discapacidad. International Journal of Developmental and Educational Psychology. Revista INFAD de Psicología, 7(1), 407-416. https://doi. org/10.17060/ijodaep.2014.n1.v7.810

Rogero-García, J. (2010). Las consecuencias del cuidado familiar sobre el cuidador: Una valoración compleja y necesaria. Index de Enfermería, 19(1). https://doi.org/10.4321/s 113212962010000100010

Salazar, A. M., Cardozo, Y., y Escobar, C. L. (2020). Carga de cuidado de los cuidadores familiares y nivel de dependencia de su familiar. Investigación en Enfermería: Imagen y Desarrollo, 22. https://doi.org/10.11144/iaveriana.ie22. cccf
Seperak Viera, R. A. (2016). Influencia De La Resiliencia En La Sobrecarga Subjetiva De Madres De Niños Con Trastorno De Espectro Autista. Revista De Psicología, 6(1), 25-48. https://35.161.26.73/index. $\mathrm{php} / \mathrm{psicologia/article/view/37}$

Vela Llauradó, E., y Suárez Riveiro, J. M. (2020). Resilience, satisfaction, and family situation with children with or without disabilities as predictors of stress in families. Ansiedad y Estres, 26(2-3), 59-66. https:// doi.org/10.1016/i.anyes.2020.03.001

Wagnild, G.M. y Young, H. M. (1993). Development and psychometric evaluation of the Resilience Scale. Journal of Nursing Measurement, 1, 165- 178. https:// cyberleninka.org/article/n/255719.pdf

Zarit, S. H., Reever, K. E., y Bach-Peterson, J. (1980). Relatives of the Impaired Elderly: Correlates of Feelings of Burden 1. The Gerontologist, 20(6), 649-655. https:// doi.org/10.1093/geront/20.6.649 


\begin{tabular}{|c|c|}
\hline \multirow{4}{*}{$\begin{array}{l}\text { ANEXO 1 } \\
\text { CUESTIONARIO PARA LAS FAMILIAS } \\
\text { (https://docs.google.com/forms// } \\
\text { d/1QSxcIWzDa6 XwG3iKMINGUrVF- } \\
\text { O2SvrigfhtQcfHSCs/edit? } \text { usp = sharing) } \\
\text { El cuestionario es totalmente anónimo y se } \\
\text { garantiza la confidencialidad de los datos. }\end{array}$} & ¿CON QUIEEN CONVIVE? \\
\hline & NÚMERODEHIJOS/AS \\
\hline & $\begin{array}{l}\text { ¿ES EL/LA CUIDADOR/A PRINCIPAL DE SUS } \\
\text { HIJOS/AS? }\end{array}$ \\
\hline & o Sí \\
\hline $\begin{array}{l}\text { A continuación, se le plantean diversas } \\
\text { preguntas que le pido que conteste con toda } \\
\text { sinceridad. }\end{array}$ & $\begin{array}{l}\text { O No } \\
\text { ¿TIENE HIJO/A CON ALGUNA DIVERSIDAD } \\
\text { FUNCIONAL? }\end{array}$ \\
\hline iMuchas gracias por su colaboración! Con & O Sí \\
\hline su participación contribuye a la investigación & O No \\
\hline $\begin{array}{l}\text { de la atención a las personas con diversidad } \\
\text { funcional. }\end{array}$ & $\begin{array}{l}\text { ¿ALGUNO DE LOS CONVIVIENTES ES UNA } \\
\text { PERSONA DEPENDIENTE? }\end{array}$ \\
\hline SEXO: & O Sí \\
\hline O Masculino & O No \\
\hline O Femenino & \\
\hline O Prefiero no decirlo & \\
\hline NIVEL DE ESTUDIOS: & \\
\hline O Primaria & \\
\hline O Secundaria & \\
\hline O Superiores & \\
\hline SITUACIÓN LABORAL: & \\
\hline O Activo & \\
\hline O Desempleo & \\
\hline $\begin{array}{l}\text { SEÑALE EL ENUNCIADO QUE, A SU } \\
\text { JUICIO, DESCRIBE MEJOR EL NIVEL } \\
\text { SOCIOECONÓMICO DE SU FAMILIA: }\end{array}$ & \\
\hline O Tenemos lo mínimo y a veces nos falta. & \\
\hline $\begin{array}{l}\text { O Dependemos de nuestro trabajo y llegamos } \\
\text { justos a fin de mes. }\end{array}$ & \\
\hline $\begin{array}{l}\text { O Tenemos ingresos suficientes para vivir sin } \\
\text { estrecheces. }\end{array}$ & \\
\hline $\begin{array}{l}\text { O Disfrutamos de una posición holgada que nos } \\
\text { permite no tener preocupaciones económicas. }\end{array}$ & \\
\hline
\end{tabular}


A CONTINUACIÓN, SE LE PRESENTA DIVERSAS PREGUNTAS EN RELACIÓN A LOS CUIDADOS DE SU/S HIJO/A(S). EN EL CASO DE TENER ALGÚN/A HIJO/A CON DIVERSIDAD FUNCIONAL CONTESTE EN RELACIÓN A ESTE/A. SIENDO 1 NUNCA Y 5 CASI SIEMPRE:

\begin{tabular}{|c|c|c|c|c|c|}
\hline & 1 & 2 & 3 & 4 & 5 \\
\hline \multicolumn{6}{|l|}{$\begin{array}{l}\text { ¿Piensa que su hija/o solicita más ayuda de la que realmente } \\
\text { necesita? }\end{array}$} \\
\hline \multicolumn{6}{|l|}{$\begin{array}{l}\text { ¿Piensa que debido al tiempo que dedica a su hijo/a ya no dispone } \\
\text { de tiempo suficiente para usted? }\end{array}$} \\
\hline \multicolumn{6}{|l|}{$\begin{array}{l}\text { ¿Se siente agobiado/a por intentar compatibilizar el cuidado de su } \\
\text { hija/o con otras responsabilidades (trabajo, familia)? }\end{array}$} \\
\hline \multicolumn{6}{|l|}{ ¿Siente vergüenza por la conducta de su hija/o? } \\
\hline \multicolumn{6}{|l|}{ ¿Se siente enfadado/a cuando está cerca de su hijo/a? } \\
\hline \multicolumn{6}{|l|}{$\begin{array}{l}\text { ¿Cree que la situación actual afecta negativamente la relación que } \\
\qquad \text { usted tiene con otros miembros de su hijo/a? }\end{array}$} \\
\hline \multicolumn{6}{|l|}{ ¿Tiene miedo por el futuro de su hijo/a? } \\
\hline \multicolumn{6}{|l|}{ ¿Piensa que su hijo/a depende de usted? } \\
\hline \multicolumn{6}{|l|}{$\begin{array}{l}\text { ¿Piensa que su salud ha empeorado debido a tener que cuidar de } \\
\text { su hija/o? }\end{array}$} \\
\hline \multicolumn{6}{|l|}{ ¿Se siente tenso/a cuando está cerca de su hija/o? } \\
\hline \multicolumn{6}{|l|}{$\begin{array}{l}\text { ¿Piensa que no tiene tanta intimidad como le gustaría debido a } \\
\text { tener que cuidar a su hija/o? }\end{array}$} \\
\hline \multicolumn{6}{|l|}{$\begin{array}{l}\text { ¿Siente que su vida social se ha visto afectada negativamente por } \\
\text { tener que cuidar de su hijo/a? }\end{array}$} \\
\hline \multicolumn{6}{|l|}{$\begin{array}{c}\text { ¿Se siente incómodo/a por distanciarse de sus amistades debido a } \\
\text { tener que cuidar de su hija/o? }\end{array}$} \\
\hline \multicolumn{6}{|l|}{$\begin{array}{l}\text { ¿Piensa que su hijo/a le considera a usted la única persona que le } \\
\text { puede cuidar? }\end{array}$} \\
\hline \multicolumn{6}{|l|}{$\begin{array}{l}\text { ¿Piensa que no tiene suficientes ingresos económicos para los gastos } \\
\text { de cuidar a su hijo/a, además de sus otros gastos? }\end{array}$} \\
\hline \multicolumn{6}{|l|}{$\begin{array}{l}\text { ¿Piensa que no será capaz de cuidar a su hijo/a por mucho más } \\
\text { tiempo? }\end{array}$} \\
\hline \multicolumn{6}{|l|}{$\begin{array}{l}\text { ¿Siente que ha perdido el control de su vida desde que nació su } \\
\text { hijo/a? }\end{array}$} \\
\hline \multicolumn{6}{|l|}{ ¿Desearía poder dejar el cuidado de su hija/o a otra persona? } \\
\hline \multicolumn{6}{|l|}{ ¿Se siente indeciso/a sobre qué hacer con su hija/o? } \\
\hline \multicolumn{6}{|l|}{ ¿Piensa que debería hacer más por su hija/o? } \\
\hline ¿Piensa que podría cuidar mejor a su hija/o? & & & & & \\
\hline $\begin{array}{l}\text { Globalmente ¿Qué grado de "carga" experimenta por el hecho de } \\
\text { cuidar a su hijo/a? }\end{array}$ & & & & & \\
\hline
\end{tabular}


A CONTINUACIÓN, SE LE PRESENTA DIVERSAS PREGUNTAS EN RELACIÓN A LOS CUIDADOS DE SU/S HIJO/A(S). EN EL CASO DE TENER ALGÚN/A HIJO/A CON DIVERSIDAD FUNCIONAL CONTESTE EN RELACIÓN A ESTE/A. SIENDO 1 NUNCA Y 5 CASI SIEMPRE:

\begin{tabular}{|c|c|c|c|c|c|c|c|}
\hline & 1 & 2 & 3 & 4 & 5 & 6 & 7 \\
\hline \multicolumn{8}{|l|}{ Es importante para mí mantenerme interesado en las cosas. } \\
\hline \multicolumn{8}{|l|}{ Dependo más de mí mismo/a que de otra personas. } \\
\hline \multicolumn{8}{|l|}{ Me mantengo interesado/a en las cosas. } \\
\hline \multicolumn{8}{|l|}{ Generalmente me las arreglo de una manera u otra. } \\
\hline \multicolumn{8}{|l|}{ Puedo estar solo/a si tengo que hacerlo. } \\
\hline \multicolumn{8}{|l|}{ El creer en mí mismo/a me permite atravesar tiempos difíciles. } \\
\hline \multicolumn{8}{|l|}{ Tengo la energía suficiente para hacer lo que debo hacer. } \\
\hline \multicolumn{8}{|l|}{ Cuando estoy en una situación difícil generalmente encuentro una salida. } \\
\hline \multicolumn{8}{|l|}{ Mi vida tiene significado. } \\
\hline \multicolumn{8}{|l|}{ Me siento orgulloso/a de haber logrado cosas en mi vida. } \\
\hline \multicolumn{8}{|l|}{ Cuando planeo algo lo hago. } \\
\hline \multicolumn{8}{|l|}{ Algunas veces me obligo a hacer cosas aunque no quiera. } \\
\hline \multicolumn{8}{|l|}{ Generalmente puede ver una situación de varias maneras. } \\
\hline \multicolumn{8}{|l|}{ Soy mi propio/a amigo/a. } \\
\hline \multicolumn{8}{|l|}{ No me lamento de las cosas por las que no puedo hacer nada. } \\
\hline \multicolumn{8}{|l|}{ Acepto que hay personas a las que no les agrado. } \\
\hline \multicolumn{8}{|l|}{ Tomo las cosas una por una. } \\
\hline \multicolumn{8}{|l|}{ Usualmente veo las cosas a largo plazo. } \\
\hline \multicolumn{8}{|l|}{ Tengo autodisciplina. } \\
\hline \multicolumn{8}{|l|}{ Siento que puedo manejar varias cosas al mismo tiempo. } \\
\hline \multicolumn{8}{|l|}{ Rara vez me pregunto cuál es la finalidad de todo. } \\
\hline \multicolumn{8}{|l|}{ Puedo enfrentar las dificultades porque las he experimentado anteriormente. } \\
\hline \multicolumn{8}{|l|}{ Soy decido/a. } \\
\hline \multicolumn{8}{|l|}{ Por lo general, encuentro algo en que reírme. } \\
\hline En una emergencia soy una persona en quien se puede confiar. & & & & & & & \\
\hline
\end{tabular}

\section{CONSENTIMIENTO INFORMADO}

Al enviar el presente formulario da su conformidad a que sus respuestas se usen de forma anónima en la realización de un trabajo de investigación y únicamente para ese propósito. Muchas gracias por su participación. 